\title{
The role of endothelin-1 and endothelin receptor antagonists in allergic rhinitis inflammation: ovalbumin- induced rat model*
}

\author{
Arzu Tatar', Muhammet Yayla², Duygu Kose², Zekai Halici², Ozgur Yoruk1, \\ Elif Polat ${ }^{3}$ \\ 'Department of Otolaryngology, Head and Neck Surgery, Ataturk University, Medical Faculty, Erzurum, Turkey \\ 2 Department of Pharmacology, Ataturk University, Medical Faculty, Erzurum, Turkey \\ ${ }^{3}$ Department of Embryology and Histology, Ataturk University, Medical Faculty, Erzurum, Turkey
}

Rhinology 54: 266-272, 2016 DOl:10.4193/Rhino15.059 *Received for publication:

March 7, 2015

Accepted: November 27, 2015

\begin{abstract}
Objective: Desloratadine is a biologically active metabolite of loratadine which is indicated for the treatment of allergic rhinitis. Bosentan is a dual endothelin receptor antagonist used to treatment of pulmonary artery hypertension (PAH). In this study, we aimed to determine the role of endothelins in allergic rhinitis (AR) and the effects of endothelin receptor antagonists in AR rat models through comparison with desloratadine.
\end{abstract}

Methods: In total, 20 adult Sprague-Dawley rats were used in this study. An ovalbumin-induced allergic rhinitis model was formed in three study groups except for the control group. Bosentan (100 mg/kg/day) was given to the bosentan-treated group for 7 days and desloratadine ( $10 \mathrm{mg} / \mathrm{kg} /$ day) was administered to the antihistaminic-treated group for 7 days. Nasal symptom scorings and histopathological examinations of the nasal tissues were carried out. Serum lgE levels and ET-1 and TNF-a mRNA expression levels were analysed. Between group comparisons for nasal symptoms, histopathological analysis, and molecular analyses were performed with a one-way ANOVA and Duncan's multiple comparison tests. Significance was accepted at $p<0.05$.

Results: Bosentan inhibited nasal symptom more significantly than desloratadine. The lgE level, ET-1 and TNF-a mRNA expression levels statistically increased in the allergic rhinitis group when compared to other groups. Conversely, the bosentan-treatment group showed a significant recovery from the same parameters. The deterioration in histopathological parameters reached the highest levels in the allergic rhinitis group. The histopathological findings were close to those of the control group in the bosentan and antihistaminic-treated group.

Conclusions: ET-1 is one of the mediators that impact AR development and ET-1 antagonists can be useful for symptom control and for decreasing allergic inflammation in AR patients.

Key words: allergic rhinitis, endothelin, endothelin receptor antagonists, bosentan, desloratadine

\section{Introduction}

Allergic rhinitis (AR) is the lgE-mediated inflammation of the nasal mucous membranes that develops after allergen inhalation. AR is characterized by a two-phase allergic reaction. In the early-phase inflammatory response, B-lymphocytes induce the synthesis of allergen-specific IgE antibodies. These IgE antibodies initiate sensitization against the allergen by binding to the receptor on mast cells and basophils. Inflammatory mediators, such as histamine, cytokine, prostaglandin, leukotriene, and platelet-activating factors are rapidly released from these sensitized cells after repeated exposure to the same allergen. These mediators lead to vasodilatation, vascular permeability increase, and mucous secretion increase, which are the characteristics of this period called the "acute phase response" and bring about symptoms in allergic rhinitis patients, such as nasal itching, sneezing, rhinorrhoea, and nasal congestion. Together 
with the rerelease of the pro-inflammatory mediators after the early-phase reaction, late-phase reactions occur in the tissue, as characterized by the accumulation of inflammatory cells, especially eosinophils ${ }^{(1)}$.

Although AR is not a life-threatening disease, it is important in terms of influencing quality of life in untreated patients. Current approaches in AR pharmacotherapy aim at decreasing inflammation and symptoms. Oral and nasal antihistamines, antileukotrienes, and nasal corticosteroids are the most commonly used agents to decrease symptoms ${ }^{(1)}$. Desloratadine is a secondgeneration antihistaminic agent and is the main metabolite of loratadine; its efficiency has been determined in AR treatment (2). Even though most of the known medications are effective in slightly- to medium-severe and intermittent diseases, they are usually insufficient for tackling severe and persistent diseases. Therefore, new research is concentrating on new agents designed to target specific immune mediators that involved in disease pathogenesis.

Endothelins (ETs) are mediators that have potent vasoconstrictor, bronchoconstrictor, and glandular secretory effects and are known for their role in immunoglobulin-E (IgE)-mediated allergic reactions ${ }^{(3-6)}$. ET has three genomic isoforms (ET-1, ET-2, and ET-3) that show an influence through two different receptors (ETA and ETB). ET-1 levels, in particular, have previously been shown to be related to increased anaphylaxis ${ }^{(6)}$, allergic asthma ${ }^{(4,5)}$, and allergic rhinitis ${ }^{(7,8)}$. ET-1 is a peptide produced by endothelial cells and has an important role in airway inflammation. ET-1 has significant pro-inflammatory effects in airways, whereas endothelin receptor antagonists mitigate its pro-inflammatory effects in animal models of airway inflammation ${ }^{(9,10)}$. Although the role of ET-1 in other allergic diseases has been extensively researched in recent years, there is a limited number of studies that examine the relationship between AR and ET-1.

Bosentan is the competitive antagonist of ET-1 in endothelin-A (ET-A) and endothelin-B (ET-B) receptors approved for treatment of pulmonary arterial hypertension ${ }^{(11)}$. The pharmacological effects of an ET-1 receptor antagonist in AR have not yet been defined. In this study, we aimed at researching the role of endothelin in AR pathogenesis in the ovalbumin (OVA) sensitized AR rat model and determining the effects of bosentan in allergic rhinitis treatment through comparison with desloratadine.

\section{Materials and methods}

Animals

This study was conducted at Ataturk University, Animal Laboratory of Medical Pharmacology, and Embryology and Histology Department. Female Sprague-Dawley rats were supplied from Ataturk University's Experimental Medical and Research Centre.
This study was approved by the Animal Ethical Committee of Veterinary Faculty (approval number; 2014-1/14). Rats were housed in standard plastic cages with sawdust bedding. The cages were placed in an air-conditioned room at $22^{\circ} \mathrm{C}$ under lighting controls (14-h-light/10-h-dark cycle). All rats had free access to standard rat food and tap water. Rats were fed a standard rat diet.

We used 20 adult female Sprague-Dawley rats (220-250 grams). Rats were randomly classified into four groups that included five rats in each group: control group (group $C$ ), allergic rhinitis group (group $A R$ ), antihistamine-treated group (group $A R+A H$ ), and bosentan-treated group (group AR+B).

\section{Preparation of OVA-induced allergic rhinitis rat model} The AR model was established according to literature-based standard protocols ${ }^{(12)}$. Specifically, 15 rats belonging to the $A R, A R+A H$, and $A R+B$ groups were sensitized with a $1 \mathrm{ml}$ i.p. injection of $30 \mathrm{mg}$ of $\mathrm{AL}(\mathrm{OH})_{3}$ and $0.3 \mathrm{mg}$ of OVA (Sigma, USA) solutions once on every second day over a period of 14 days (totalling 7 injections). Then, sensitization continued via nasal challenge (by putting drops in bilateral nasal cavities) with $20 \mu \mathrm{l}$ $10 \%$ OVA ones a day from the 15 th day to the 21 st day. All rats in the $\mathrm{AR}+\mathrm{AH}$ group orally received $10 \mathrm{mg} / \mathrm{kg}$ desloratadine dissolved in saline solution, and the $A R+B$ group received $100 \mathrm{mg} /$ $\mathrm{kg}$ bosentan administered orally in $1 \mathrm{ml} \mathrm{PBS}$ and $1 \mathrm{ml}$ distilled water once every 7 days at the same time with nasal challenge. The $C$ group and the AR group were given a saline solution alone according to the same schedule. The experimental model was very well tolerated by the animals.

\section{Evaluation of nasal symptoms}

On day 21 of the study, rats were placed in an observation cage for about $10 \mathrm{~min}$ for acclimatization before experimentation began. After nasal instillation of $20 \mu \mathrm{l} 10 \%$ OVA into bilateral nasal cavities, the animals were placed in observation cages (one animal/cage), and the numbers of sneezes and nasal rubbing movements were counted for 30 min by four blinded observers who were unaware of the sample's identity ${ }^{(13)}$. The mean of nasal symptom score of four observers was taken for each rat. Next, blood samples were taken from each rat by cardiac puncture under anaesthesia using isoflurane; the rats were then killed. A serum was prepared and frozen at $-80^{\circ} \mathrm{C}$ prior to analysis. Nasal tissue was immediately removed from all the rats at the end of the study for histopathological changes and molecular analyses.

\section{Immunoglobulin E measurements in serum}

OVA specific lgE levels in serum were measured in duplicate using a highly sensitive enzyme-linked immunosorbent assay (Sunred, China) according to the manufacturer's instructions (201-11-4050) for kits designed specifically for rats. 


\section{Molecular analyses}

The samples for molecular analyses were immediately stored at $-80^{\circ} \mathrm{C}$. Before storage, all of the tissue samples from each rat were first perfused with PBS/heparin and then ground into liquid nitrogen with a TissueLyser II grinding Jar Set.

\section{Total RNA extraction and CDNA synthesis}

The nasal cavity tissues $(20 \mathrm{mg}$ ) were stabilized in an RNA stabilization reagent (RNAlater, Qiagen) and then disrupted with the TissueLyser II (Qiagen) (2×2 min.). The total RNA was purified using an RNeasy Mini Kit (Qiagen) according to the manufacturer's instructions. The RNA samples were reverse-transcribed into complementary DNA with a high-capacity cDNA reverse transcription kit (Applied Biosystems). The total RNA (10 $\mu \mathrm{L})$ was treated with $2 \mu \mathrm{L}$ of 10X RT buffer, $0.8 \mathrm{~mL}$ of $25 \mathrm{X}$ dNTP mix, $2 \mu \mathrm{L}$ of 10X RT random primers, $1 \mu \mathrm{L}$ multiscribe reverse transcriptase, and $4.2 \mu \mathrm{L}$ of DEPC-H2O. Reverse transcription was carried out at $25^{\circ} \mathrm{C}$ for $10 \mathrm{~min}$, followed by $120 \mathrm{~min}$ at $37^{\circ} \mathrm{C}$, and finally $85^{\circ} \mathrm{C}$ for 5 min using a Veriti 96 Well Thermal Cycler (Applied Biosystems). The cDNA concentration and quality were assessed and quantified using the Epoch Spectrophotometer System and Take3 Plate (Biotek).

\section{Relative quantification of gene expression}

Relative Endothelin 1 and TNF-a expression analysis were performed with StepOne Plus Real Time PCR System technology (Applied Biosystems) using cDNA synthesized from nasal cavity tissue RNA. Further, qPCR was run using the Primer Perfect Probe Mix and Taqman Probe-based technology (Primer Design Ltd., Southampton, UK). The results were expressed as a relativefold compared to the control group. The expression of $\beta$-actin in each tissue sample was used as an endogenous control. The primers and probes for $\beta$-actin were designed by Primer Design, Southampton, UK. For each tissue, triplicate determinations were performed in a 96-well optical plate for both targets using $9 \mu \mathrm{L}$ of cDNA (100 ng), $1 \mu \mathrm{L}$ of Primer Perfect Probe mix, and 10 $\mu \mathrm{L}$ of QuantiTect Probe PCR Master mix (Qiagen, Hilden, Germany) in each $20 \mu \mathrm{L}$ reaction. The plates were heated for $2 \mathrm{~min}$ at $50^{\circ} \mathrm{C}$ and $10 \mathrm{~min}$ at $95^{\circ} \mathrm{C}$, and subsequently 40 cycles of $15 \mathrm{~s}$ at $94^{\circ} \mathrm{C}$ and $60 \mathrm{~s}$ at $60^{\circ} \mathrm{C}$ were applied. Using the $2-\Delta \Delta \mathrm{Ct}$ method, all data are expressed as the fold-change in the expression of the ET-1 and TNF-a compared to the expression in the control group.

\section{Histopathological examination}

The tissues reserved for histopathological examination were rapidly fixed in $10 \%$ buffered formalin for $24 \mathrm{~h}$. After fixation, a tissue sample was routinely processed and embedded in paraffin. For routine histological examination, $5 \mu \mathrm{m}$-thick sections were cut as paraffin-embedded tissue samples. After $5 \mu$ m-thick sections of tissue were obtained onto positively charged slides

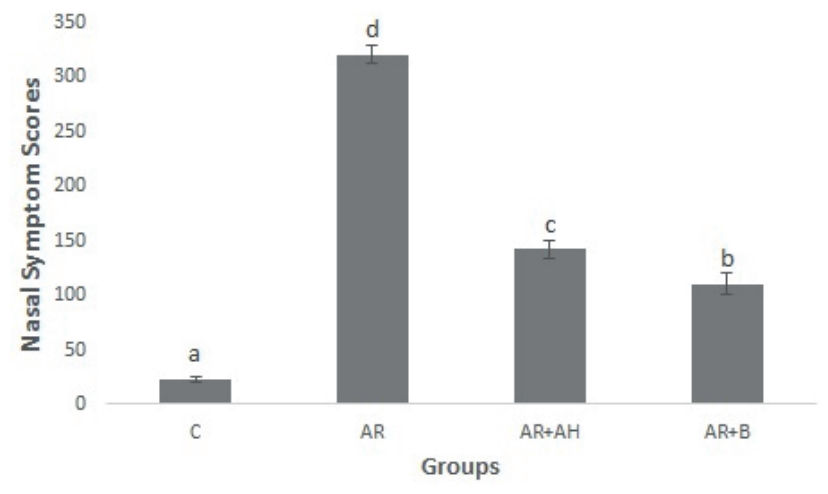

Figure 1. On the 21 th day of the study, total nasal symptom scores were assessed as mean of each group. Between-group comparisons were performed with a one-way ANOVA and Duncan's multiple comparison tests. Significance was accepted at $p<0.05$. The results were presented as mean $\pm S D$. The SD of each group was remarked on the bar of the group. The bars in the different serial shown by the different letter significantly differ from each other at a level of 5\%. The lines with closed two ends on the bars showed the Standard Deviations. $C=$ Control; $A R=A$ llergic Rhinitis; $\mathrm{AR}+\mathrm{AH}=$ Allergic Rhinitis+Antihistamine-treated group; $\mathrm{AR}+\mathrm{B}=$ Allergic Rhinitis+Bosentan treated group.

and then deparaffinization and rehydration, two sections of each rat were stained with Mayer's hematoxylin-eosin and toluidin blue. All sections were examined and photographed by a light photomicroscope (Nikon Eclipse E600).

Histopathological evaluations of sections of each rat were semiquantitatively scored. The $100 \mu^{2}$ area was distinguished by means of a $\mu \mathrm{m}$ for the X10 magnification. In each section of each rat, the parenchyma and stroma parts of tissues were counted at X10 objective in 5 randomly chosen microscopic areas and the arithmetic mean was semi-quantitatively scored. The scoring was determined as follows; absent $=0$, mild $=1$, moderate $=$ 2 , and severe $=3$. The rates of cilia losses, inflammatory cells, goblet cells, mast cells, vascular congestions, and vascular dilatations were scored in histopathological assessment.

\section{Statistical analyses}

The IBM SPSS, Version 20.0 (IBM Corporation, Somers, NY, USA) was used for the molecular analysis, nasal symptoms, and histopathological analysis. The results are presented as mean \pm standard deviation (SD). Between-group comparisons for molecular analyses, nasal symptoms, and histopathological analysis were performed with a one-way ANOVA and Duncan's multiple comparison tests. Significance was accepted at $p<0.05$.

\section{Results}

Nasal symptom score

The mean of each group has been calculated (Figure 1). Bosentan extract more significantly inhibited the nasal symptom 


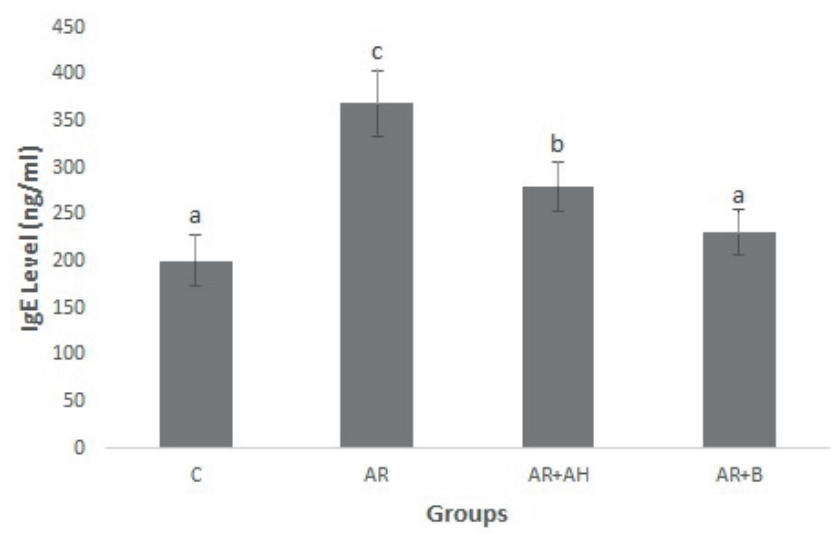

Figure 2. Difference in IgE levels between study groups; group AR have the highest IgE level. Between-group comparisons were performed with a one-way ANOVA and Duncan's multiple comparison tests. Significance was accepted at $p<0.05$. The results were presented as mean $\pm S D$. The $S D$ of each group was remarked on the bar of the group. $C$ group (a) did not show any difference statistically from $A R+B$ group (a). But $C$ group (a) showed a difference from AR+AH group (b). The bars in the different serial shown by the different letter significantly differ from each other at a level of $5 \%$. The lines with two closed ends on the bars showed the Standard Deviations. $C=$ Control; $A R=A l l e r g i c$ Rhinitis; $A R+A H=A l l e r g i c$ Rhinitis+Antihistamine-treated group; $A R+B=A l l e r g i c$ Rhinitis+Bosentan treated group.

than desloratadine $(p<0.05)$ (Figure 1).

\section{IgE level in serum}

It was observed that the IgE level in the AR group statistically increased when compared to healthy groups in the study. Further, the IgE level is seen to have significantly recovered in the groups in which we applied bosentan and antihistamine therapy (Figure 2).

\section{ET-1 and TNF-a mRNA expression}

We investigated the level of ET-1 and TNF-a mRNA expressions in the nasal cavity tissue of the rats by real-time PCR. ET-1 gene expression increased in the AR group compared to control groups $(p<0.05)$. Bosentan and desloratadine showed a significantly down-regulatory effect on ET-1 mRNA expression in AR rats, respectively (Figure 3). Our results also suggest that bosentan has a higher down regulatory effect than desloratadine. Compared to the control group, TNF-a mRNA level was significantly higher in the AR group ( $p<0.05)$ (Figure 4). Bosentan and desloratadine were found to reduce the level of TNF-a expression in the nasal cavity tissues of rats.

\section{Histopathological results}

Light microscopic results are in depicted in Figure 5 and Table 1. Findings that belong to group C: Nasal respiratory mucosa was found to be normal when the nasal cavity sections of the control group were evaluated histopathologically. Basal membranes

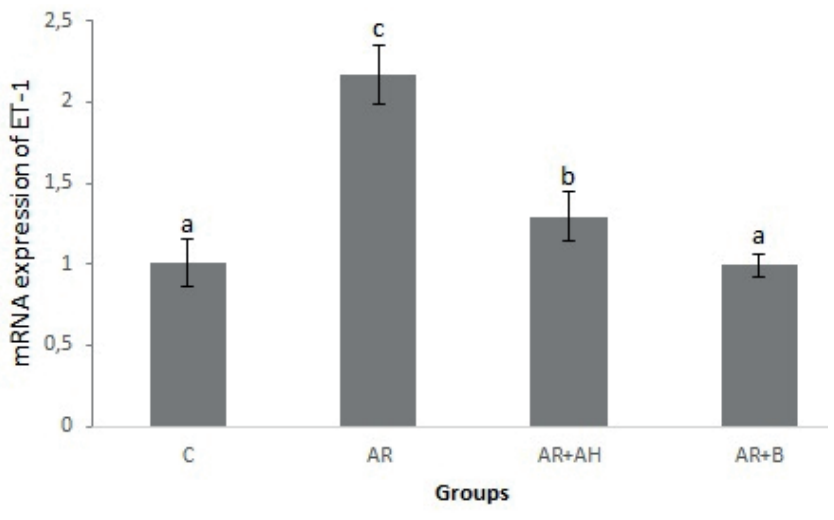

Figure 3. Difference in ET-1 mRNA expression levels between study groups; ET-1 expression levels of group $C$ and group $A R+B$ close to each other. The results were presented as mean \pm standard deviation (SD). The SD of each group was remarked on the bar of the group. Between-group comparisons were performed with a one-way ANOVA and Duncan's multiple comparison tests. Significance was accepted at $p<0.05$. The bars in the different serial shown by the different letter significantly differ from each other at a level of $5 \%$. The lines with two closed ends on the bars showed the Standard Deviations. $C=$ Control; $A R=$ Allergic Rhinitis; $\mathrm{AR}+\mathrm{AH}=$ Allergic Rhinitis+Antihistamine-treated group; $\mathrm{AR}+\mathrm{B}=$ Allergic Rhinitis+Bosentan treated group.

and stratified pseudo-epitheliums were observed as healthy; intraepithelial glands and goblet cells indicated normal sizes and numbers.

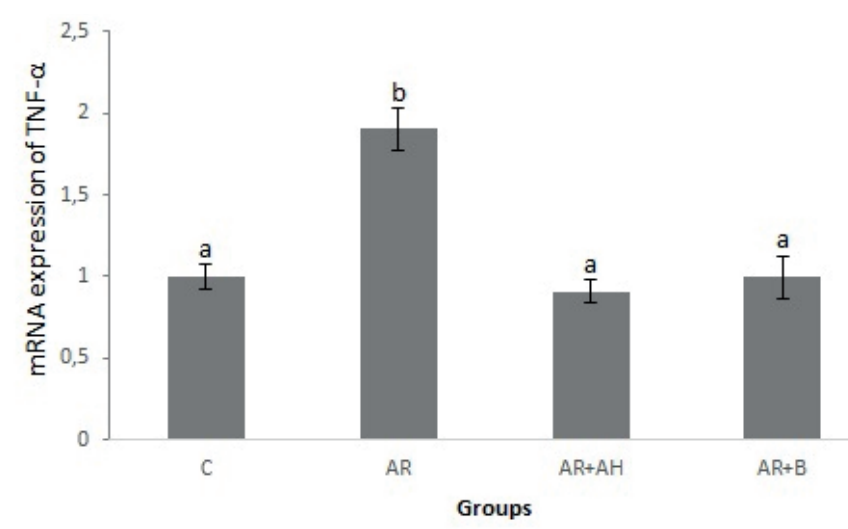

Figure 4. Difference in TNF-a mRNA expression levels; expression levels of TNF-a were not differ in the group $C, A R+A H$, and $A R+B$. Betweengroup comparisons were performed with a one-way ANOVA and Duncan's multiple comparison tests. Significance was accepted at $p<0.05$. The results were presented as mean \pm standard deviation (SD). The SD of each group was remarked on the bar of the group. The bars in the different serial shown by the different letter significantly differ from each other at a level of $5 \%$. The lines with two closed ends on the bars showed the Standard Deviations. $C=$ Control; $A R=$ Allergic Rhinitis; $A R+A H=$ Allergic Rhinitis+Antihistamine-treated group; $A R+B=A l l e r g i c$ Rhinitis+Bosentan treated group. 

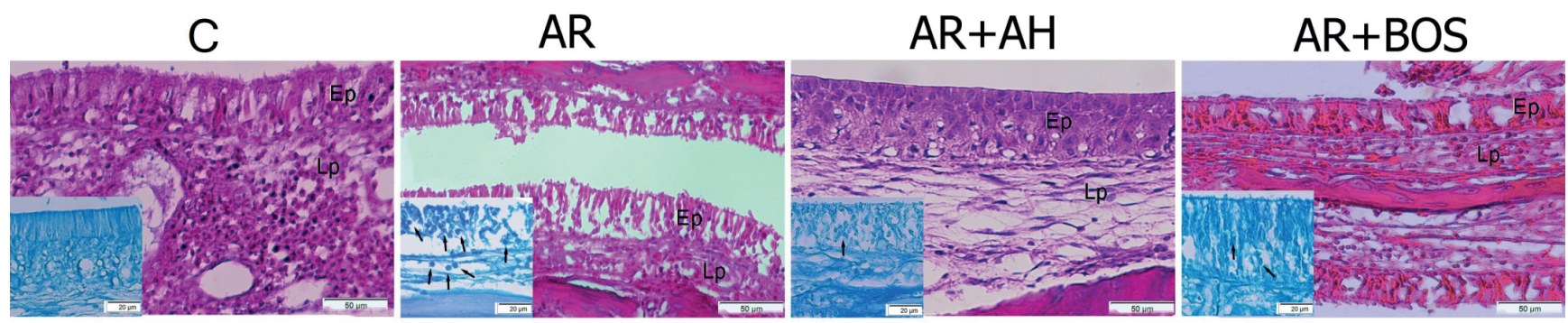

Figure 5. Histopathological sections of all the experiment's groups have been presented together: Big square; $\mathrm{H} \& \mathrm{E}$, small square; toluidine blue. Section thickness; $5 \mu \mathrm{m}$. C=Control; $\mathrm{AR}=$ Allergic Rhinitis; $\mathrm{AR}+\mathrm{AH}=\mathrm{Allergic}$ Rhinitis+Antihistamine-treated group; $\mathrm{AR}+\mathrm{BOS}=\mathrm{Allergic}$ Rhinitis+Bosentan treated group. Ep; respiratory epithelium, Lp; lamina propria, arrow (); leucocyte.

Findings that belong to group AR: Nasal respiratory epithelium thickening, edema, inflammatory cells, and cilia losses were observed in the nasal cavity sections of the group with allergic rhinitis. Degeneration and edema were detected in the mucous membrane, and inflammatory cell infiltration was found in the lamina propria. Dilated and congested areas were encountered in vascular areas. Hyperplasia was detected in the seromucous glands of the lamina propria. Adhered neutrophil in the sections stained with toluidine blue had a metachromatic appearance. Findings that belong to group $\mathrm{AR}+\mathrm{AH}$ : It was seen that the mucosa oedema of the rats, which had allergic rhinitis and desloratadine treatment, was less than the group AR. While damaged areas were observed in the basal membrane of the nasal respiratory epithelium, cilia losses continued on the epithelial apical surface. When compared to the group AR, a considerable polymorphonuclear infiltration decrease and the existence of fibroblast and collagen fiber synthesis were detected in the lamina propria. In sections stained with toluidine blue dye, there were rare neutrophil cells in the intraepithelial area.

Findings that belong to group AR+B: The nasal respiratory epithelium in the nasal cavity sections of group AR, which had the bosentan application, was similar to that of the control group. An increase was seen in cilia on the epithelial apical surface compared to the group with AR. Another increase was observed in the number of goblet cells. Considering the lamina propria inflammatory cell infiltration, a decrease was detected compared to the group with AR. Specifically, whereas inflammatory cell infiltration decreased compared to the AR group, an increase was observed in comparison to the AR+AH group. However, collagen density was less than group $\mathrm{AR}+\mathrm{AH}$. Rare areas of inflammation were encountered in the sections stained with toluidine blue dye.

\section{Discussion}

The nasal epithelium is the area where cytokine and other pro-inflammatory mediators are synthesized and secreted, and therefore has an active role in allergic inflammation. ETs are secreted from submucosal glands in the nasal epithelial and endothelial cells in the nose. ETs enable epithelial permeability control and contribute to the regulation of nasal mucosa haemostasis. Additionally, they also take part in the regulation of mucociliary activity in maxillary sinuses, nasal glandular secretion, pro-inflammatory mediator release, and inflammatory cell recruitment ${ }^{(14-17)}$. ETs, especially ET-1, might have a role in

Table 1. The results of semi-quantitative histopathological analysis of the submandibular glands.

\begin{tabular}{cccccccc} 
Groups & No. of rats & CL & IC & GS & MS & VC & VD \\
\hline Control & 5 & $0.07 \pm 0.04^{\mathrm{c}}$ & $1.01 \pm 0.20^{\mathrm{c}}$ & $0.17 \pm 0.08^{\mathrm{b}}$ & $1.12 \pm 0.15^{\mathrm{b}}$ & $0.25 \pm 0.09^{\mathrm{b}}$ & $0.39 \pm 0.04^{\mathrm{c}}$ \\
\hline $\mathrm{AR}$ & 5 & $3.02 \pm 0.14^{\mathrm{a}}$ & $3.94 \pm 0.53^{\mathrm{a}}$ & $2.92 \pm 0.08^{\mathrm{a}}$ & $2.93 \pm 0.08^{\mathrm{a}}$ & $3.03 \pm 0.12^{\mathrm{a}}$ & $2.08 \pm 0.18^{\mathrm{a}}$ \\
\hline $\mathrm{AR}+\mathrm{AH}$ & 5 & $3.01 \pm 0.26^{\mathrm{a}}$ & $1.04 \pm 0.14^{\mathrm{c}}$ & $1.02 \pm 0.16^{\mathrm{b}}$ & $1.05 \pm 0.14^{\mathrm{b}}$ & $0.12 \pm 0.07^{\mathrm{b}}$ & $0.08 \pm 0.05^{\mathrm{c}}$ \\
\hline $\mathrm{AR}+\mathrm{B}$ & 5 & $2.00 \pm 0.18^{\mathrm{b}}$ & $2.02 \pm 0.18^{\mathrm{b}}$ & $1.29 \pm 0.11^{\mathrm{b}}$ & $1.05 \pm 0.12^{\mathrm{b}}$ & $0.11 \pm 0.04^{\mathrm{b}}$ & $1.00 \pm 0.13^{\mathrm{b}}$ \\
\hline
\end{tabular}

Values with the same superscript letters in the same column are not statistically different $(p<0.05)$. Results are mean $\pm S D$ of the mean. AR $=$ allergic rhinitis; $A R+A H=$ allergic rhinitis+antihistamine-treated; $A R+B=$ allergic rhinitis+bosentan-treated, $C L ;$ cilia losses, IC; inflammatory cell, GC; goblet cell, MC; mast cell, VC; vascular congestion, VD; vascular dilatation 
allergic rhinitis pathophysiology by stimulating nasal glandular secretions, increasing the mucociliary activity and the release of pro-inflammatory mediators, and also by causing inflammatory cell chemotaxis ${ }^{(14)}$.

There is a limited number of studies in the literature examining the role of ET-1 in the inflammatory disease pathogeneses of the upper airways. ET-1 mRNA expression and (ECE)-1 co-expression increase in the glands and epithelial cells of human nasal mucosa with chronic inflammation ${ }^{(7)}$. ETs expression increase in the inflammatory cells and glands of nasal polyps and allergic mucosa ${ }^{(17)}$. A study conducted on patients with a birch pollen allergy found that ET-1 levels in nasal lavage fluid was higher in allergic patients than in the control group after the allergen challenge, and that ET-1 might have a role in the seasonal allergic rhinitis pathogenesis ${ }^{(8)}$. Riccio et al. showed that a more clear-cut increase emerged in the nasal symptoms and glandular product secretions of allergic individuals when nasal provocation was applied with ET-1 to allergic and non-allergic individuals ${ }^{(18)}$. These findings indicate the role of allergic inflammation in excessive response of the nasal mucosa against ET-1.

In our study, ET-1 expression in the nasal tissues of OVA-sensitized rats was significantly higher than in the control group. A parallel increase in nasal symptom scores was observed in ET-1 expression levels in all the groups. Moreover, the ET-1 level and allergic rhinitis symptom scores were shown to be lower in the bosentan-treated group than in the antihistaminic-treated group. These results indicate that ET-1 has a role in allergic rhinitis pathogenesis and the ET-1 receptor blockage may be useful for the control of allergic rhinitis symptoms.

There are no studies available in the literature, as research is focused on the effects of endothelin receptor blockers on allergic rhinitis. However, there are researches that reveal that ET receptor blockers are effective in treatment of allergic reactions like anaphylaxis and allergic asthma ${ }^{(4,15,19)}$. Studies do exist in literature reporting that ETA antagonists might decrease the mast cell activation activated by endogenous ET-1 during an allergic reaction ${ }^{(19)}$ and the inhibition of ETA receptors or combined ETA and ETB receptors in antigen-induced lung inflammation lead to a significant decrease in airway inflammation ${ }^{(15)}$. In addition, two recent studies that implemented an asthma murine model showed that ET receptor antagonists were effective in inhibiting the acute allergic response ${ }^{(20,21)}$.

ETs were claimed to cause the influx of inflammatory cells by inducing pro-inflammatory mediator release and functioning as chemo-attractant ${ }^{(4,5,22)}$ and in this way contribute to the development of long-term inflammatory changes in nasal respiratory mucosa independently from the original stimulus ${ }^{(23,24)}$. The fact that the endothelin expression levels and inflammatory cell infiltration of allergic rhinitis group rats were higher than those of the control group, as seen in the histological results of our study, supports this claim. Besides, the approach of inflammatory cell infiltration and other histopathological findings in the bosentantreated allergic rhinitis group compared to the control group indicate that late-phase reactions can be decreased by using ET-1 receptor blockers.

Many interactions between ET-1 and other cytokines essential in allergic disorders have been described. Binding of ET-1 to ET receptors can induce strong and rapid degranulation of mast cells and causes the secretion of cytokines ${ }^{(22,25)}$. Tumour necrosis factor-alpha (TNF-a), which is one of these cytokines, led to trans-epithelial migration of neutrophils, activated the secretion of various cytokines, and could be related to acquired airway hypersensitivity ${ }^{(26)}$. Treatment of the rats with the ET receptor antagonist bosentan resulted in a substantial decrease in the concentrations of TNF- $a$ and ET- ${ }^{(4)}$. Current studies assert that the inhibition of cytokine activations may be useful for the prevention of inflammation, and therefore, TNF-a inhibition may also be useful for the prevention of allergic inflammation. This study indicated that ovalbumin specific lgE antibody and TNF- $a$ expression level in the bosentan-treated group, which was the highest level in the allergic rhinitis group, was closer to the values of the control group compared to the desloratadine treatment group. Relying on these results, we are of the opinion that the ET-1 antagonistic effect can be influential in the treatment of allergic inflammation by inhibiting the TNF-a production, and this in turn can constitute a foundation for alternative approaches in AR treatment in the future.

Desloratadine has linear pharmacokinetics over the $5-20 \mathrm{mg}$ dose range ${ }^{(27)}$. It is usually use at $5-10 \mathrm{mg} / \mathrm{kg} /$ day dosage in allergic disease. We used desloratadine at $10 \mathrm{mg}$ dosage in this study. Bosentan have been used at $125-250 \mathrm{mg} / \mathrm{kg} /$ twice day for treated of pulmonary hypertension and it may cause the liver dysfunction. Bosentan have some adverse effects on hematologic, neurologic, cardiovascular, respiratory, and gastrointestinal systems. Hepatotoxicity may be the most important factor that restricts the use of bosentan for treatment at allergic rhinitis. Liver dysfunction that result from use of bosentan evolves as linked to duration and dose of exposure. It has been reported a significantly higher incidence of high liver enzymes in the bosentan $250 \mathrm{mg}$ twice daily group compared to the placebo group but there was no significant difference in the bosentan $125 \mathrm{mg}$ twice daily group ${ }^{(28)}$. Liver dysfunction recovers with either no intervention, dose reduction, or drug discontinuation. We detected that bosentan take effect in allergic rhinitis at the lower doses ( $100 \mathrm{mg} / \mathrm{kg} /$ day) than the doses used for pulmonary hypertension. The new studies are necessary to indicate 
the effect and toxicity of bosentan at the smaller doses at use in allergic rhinitis therapy.

\section{Conclusion}

Based on these data, we presume the following hypothesis for the role of ET-1 in the occurrence of eosinophilic airway inflammation and ET-1 antagonists such as bosentan may have potential benefits for the patients with allergic rhinitis inflammation and control of allergic rhinitis symptoms, although there is need for further investigation.

\section{Acknowledgements}

We thanks to Hasan Turkez, MD ,PhD from Erzurum Technical University for statistical analyses.

\section{Authorship contribution}

Arzu Tatar, corresponding author, conception and design of the study, analysis and interpretation of data, and wrote the manuscript, Muhammet Yayla, conception and design and acquisition and analysis of biochemical data, Duygu Kose, acquisition of data, and analysis and interpretation of data, Zekai Halici, conception and design, acquisition, analysis and interpretation of data, manuscript preparation, Ozgur Yoruk, conception and design, analysis and interpretation of data, manuscript preparation and approval, Elif Polat, conception and design, acquisition and analysis of histopathological and stereological data, and manuscript writing.

\section{Conflict of interest}

The authors declare no conflict of interest.

\section{References}

1. Castillo J, Dimov V. Investigational drugs for the treatment of allergic rhinitis. Expert Opin Investig Drugs 2014; 23:823-836.

2. Gonzalez-Nunez V, Valero A, Mullol J. Safety evaluation of desloratadine in allergic rhinitis. Expert Opin Drug Saf 2013; 12:445-453.

3. Kawanabe Y, Nauli SM. Endothelin. Cell Mol Life Sci 2011; 68:195-203.

4. Finsnes F, Lyberg $T$, Christensen G, Skjønsberg $\mathrm{OH}$. Effect of endothelin antagonism on the production of cytokines in eosinophilic airway inflammation. Am J Physiol Lung Cell Mol Physiol 2001; 280:L659-665

5. Finsnes F, Christensen G, Lyberg T, Sejersted $\mathrm{OM}$, Skjønsberg $\mathrm{OH}$. Increased synthesis and release of endothelin-1 during the initial phase of airway inflammation. Am J Respir Crit Care Med 1998; 158:1600-1606.

6. Shigematsu T, Miura S, Hirokawa M et al. Endothelins promote egg albumininduced intestinal anaphylaxis in rats. Gastroenterology 1998; 115:348-356.

7. Furukawa K, Saleh D, Bayan F et al. Co-expression of endothelin-1 and endothelin-converting enzyme-1 in patients with chronic rhinitis. Am J Respir Cell Mol Biol 1996; 14:248-253.

8. Gawlik R, Jawor B. Endothelin-1 in nasal lavage fluid of allergic rhinitis patients-new mediator of allergic rhinitis. Otolaryngol Pol 2007; 61: 567-571.

9. Araz O, Demirci E, Yilmazel Ucar E et al Comparison of reducing effect on lung injury of dexamethasone and bosentan in acute lung injury: an experimental study. Multidiscip Respir Med 2013; 8:74

10. Yahiaoui L, Villeneuve A, ValderramaCarvajal H, Burke F, Fixman ED. Endothelin-1 regulates proliferative responses, both alone and synergistically with PDGF, in rat tracheal smooth muscle cells. Cell Physiol Biochem 2006; 17:37-46.

11. Weber C, Gasser R, Hopfgartner G Absorption, excretion, and metabolism of the endothelin receptor antagonist bosen- tan in healthy male subjects. Drug Metab Dispos 1999; 27:810-815.

12. Wang W, Zheng M. Nuclear factor kappa B pathway down-regulates aquaporin 5 in the nasal mucosa of rats with allergic rhinitis. Eur Arch Otorhinolaryngol 2011; 268:73-81

13. Wang M, Zhang W, Shang J, Yang J, Zhang $L$, Bachert C. Immunomodulatory effects of IL-23 and IL-17 in a mouse model of allergic rhinitis. Clin Exp Allergy 2013; 43:956-966.

14. Mullol J, Picado C. Endothelin in nasal mucosa: role in nasal function and infammation. Clin Exp Allergy 2000; 30:172-177.

15. Casasco A, Benazzo M, Casasco M et al Occurrence, distribution and possible role of the regulatory peptide endothelin in the nasal mucosa. Cell Tissue Res 1993: 274:241247.

16. Mullol J, Baraniuk JN, Picado C, Shelhamer $\mathrm{JH}$. Endothelin and the epithelium. In: Goldie RG, Hay DWP, eds. Pulmonary Actions of the Endothelins. Basel: BirkhaĖuser-Verlag AG, 1999; 145-166.

17. Ohkubo K, Ohnishi M, Yokoshima K, Takizawa R, Okuda M, Kaliner MA. Study of endothelin: distribution in the airway and release from nasal epithelial cells. Arerug 1994; 43:448-457.

18. Riccio MM, Reynolds CJ, Hay DWP, Proud D. Effect of intranasal administrations of endothelin-1 to allergic and nonallergic individuals. Am J Respir Crit Care Med 1995; 152:1757-1764.

19. Metz M, Schafer B, Tsai M, Maurer M, Galli SJ. Evidence that the endothelin A receptor can enhance lgE-dependent anaphylaxis in mice. J Allergy Clin Immunol 2011; 128:424426

20. Landgraf RG, Jancar S. Endothelin A receptor antagonist modulates lymphocyte and eosinophil infiltration, hyperreactivity and mucus in murine asthma. Int Immunopharmacol 2008; 8:1748-1753.

21. Kassuya CA, Rogerio AP, Calixto JB. The role of $E T(A)$ and $E T(B)$ receptor antagonists in acute and allergic inflammation in mice.
Peptides 2008; 29:1329-1337

22. Cunningham ME, Huribal M, Bala RJ, McMillen MA. Endothelin-1 and endothelin-4 stimulate monocyte production of cytokines. Crit Care Med 1997; 25:958-964.

23. Elferink JG, de Koster BM. Endothelininduced activation of neutrophil migration. Biochem Pharmacol 1994; 48: 865-871.

24. Helset E, Ytrehus K, Tveita T, Kjaeve J, Jorgensen L. Endothelin-1 causes accumulation of leukocytes in the pulmonary circulation. Circ Shock 1994; 44:201-209.

25. Matsushima $H$, Yamada $N$, Matsue $H$, Shimada S. The effects of endothelin-1 on degranulation, cytokine, and growth factor production by skin-derived mast cells. Eur J Immunol 2004; 34:1910-1919.

26. $\mathrm{Xu}$ J, Zhong NS. The interaction of tumor necrosis factor-alfa and endothelin-1 in pathogenic models of asthma. Clin Exp Allergy 1997; 27:568-573.

27. Gupta S, Banfield C, Affrime M Desloratadine demonstrates dose proportionality in healthy adults after single doses. Clin Pharmacokinet 2002; 41:1-6

28. Aversa M, Porter S, Granton J. Comparative safety and tolerability of endothelin receptor antagonists in pulmonary arterial hypertension. Drug Saf 2015; 38:419-435.

Arzu Tatar, MD

Ataturk Uni. Tıp Fak. Kulak-Burun-

-Bogaz AD

25240-Erzurum

Turkey

Mobile: +90 535 445-1939

E-mail: berke327@mynet.com 